\title{
KÄHLER-RICCI FLOW WITH DEGENERATE INITIAL CLASS
}

\author{
ZHOU ZHANG
}

\begin{abstract}
In an earlier joint work with X. X. Chen and G. Tian (2011), we introduced the weak Kähler-Ricci flow for various geometric motivations. In this current work, we give further consideration to setting up the weak flow by allowing the initial class to be not necessarily Kähler. It's shown that the construction is compatible with the earlier construction in the Kähler case. We also discuss the convergence as $t \rightarrow 0^{+}$, which is of great interest in this topic, and provide related motivation.
\end{abstract}

\section{Motivation AND SET-UP OF WEAK FLOW}

The Kähler-Ricci flow, the complex version of Ricci flow, has been the subject of intensive study over the last twenty years. In [18] and more recently [17, G. Tian proposed the intriguing program of constructing globally existing (weak) KählerRicci flow with canonical (singular) limit at time infinity and applying it to the study of algebraic manifolds (and even Kähler manifolds in general). It can be viewed as the analytic version of the famous Minimal Model Program in algebraic geometry.

In general, people should expect the classic smooth Kähler-Ricci flow to encounter singularity at some finite time which is completely decided by cohomology information according to the optimal existence result in 20. Based on the history and success and in cases regarding Ricci flow, surgeries for the underlying manifold should be expected. For the Kähler-Ricci flow, we naturally expect the surgery to have flavours from algebraic geometry.

A simple example would be surfaces of general type. One only needs the blowingdown of $(-1)$-curves when applying the general construction at the end of 3 ] to push the flow through the finite time of singularities, where the measure restriction is actually not so involved. This will be explained later in Example 4.4. The degenerate class at the singularity time would become Kähler for the new manifold because those $(-1)$-curves causing the cohomology degeneration would eventually be crushed to points.

Things can get significantly more complicated for higher dimensions. For example, a standard procedure called flip is introduced in the algebraic geometry context, which is of great importance for the algebraic Minimal Model Program. Simply speaking, one needs to blow up the manifold and then perform a different

Received by the editors January 6, 2012.

2010 Mathematics Subject Classification. Primary 53C44; Secondary 14E30, 58J35.

Key words and phrases. Geometric evolution equation, minimal model program.

The author was supported in part by NSF 0904760 and ARC Discovery Project DP110102654. 
blowing-down process. Naturally, we should expect the transformation of the degenerate class to not be Kähler. In this note, we confirm that this is not a problem under the assumption that formally the Kähler-Ricci flow is instantly taking the class into the Kähler cone of the new manifold. As in [3], short time existence is what it's all about.

Plan of this work. For the rest of Section 1, we provide the details of the weak flow construction using approximation. In Section 2, we justify regularity of the thus defined weak flow away from the initial time. In Section 3, we show that the construction here is compatible with that in [3, i.e. they give the same weak flow when the initial class is Kähler. In Section 4 , we describe the situation when $t \rightarrow 0^{+}$ in various cases of geometric interest. Finally, we conclude in Section 5 with some remarks.

In the following, we set up the weak flow following the same idea as in 3 , with a priori very little regularity in either time or space direction. To begin with, we specify the way the problem is considered.

Let $X$ be a closed Kähler manifold with $\operatorname{dim}_{\mathbb{C}} X=n \geqslant 2$. We consider the following version of Kähler-Ricci flow over $X$ :

$$
\frac{\partial \widetilde{\omega}_{t}}{\partial t}=-\operatorname{Ric}\left(\widetilde{\omega}_{t}\right)-\widetilde{\omega}_{t}, \widetilde{\omega}_{0}=\omega_{0} .
$$

More importantly, the initial data is weak as follows:

$$
\omega_{0}=\omega+\sqrt{-1} \partial \bar{\partial} v,
$$

where $\omega$ is a real, smooth, and closed $(1,1)$-form with $[\omega]$ being nef. (i.e. numerically effective, in other words, on the boundary of the Kähler cone of $X$ ), and $v \in$ $P S H_{\omega}(X) \cap L^{\infty}(X)$ where $v \in P S H_{\omega}(X)$ means $\omega+\sqrt{-1} \partial \bar{\partial} v \geqslant 0$ weakly (i.e. in the sense of distribution).

Remark 1.1. This version of Kähler-Ricci flow is simply a time-metric scaled version of $\frac{\partial \widetilde{\omega}_{t}}{\partial t}=-\operatorname{Ric}\left(\widetilde{\omega}_{t}\right)$, which makes no difference to our interest here. In fact, the discussion is valid for other unconventional Kähler-Ricci type flows as discussed in [20] and 22].

The Main Assumption for this work is the following: suppose $\left[\omega_{0}\right]=[\omega]$ is on the boundary of the Kähler cone of $X$ (i.e. being nef.), which sits in the cohomology space $H^{1,1}(X, \mathbb{C}) \cap H^{2}(X, \mathbb{R})$, and the ray starting from $[\omega]$ and in the direction towards the canonical class of $X, K_{X}$, enters the Kähler cone instantly. Clearly, there is no need for $K_{X}$ to be Kähler for this to be the case.

There are other motivations for studying this case besides defining weak flow to carry out Tian's program as described before. In general, there is expectation that the general geometric and analytic properties of the Kähler-Ricci flow should be decided largely by the cohomology information from the formal ODE consideration in $H^{1,1}(X, \mathbb{C}) \cap H^{2}(X, \mathbb{R})$. The main theorem below would strengthen this philosophical point of view.

Theorem 1.2. Under the Main Assumption above, one can define a unique weak Kähler-Ricci flow from the Approximation Construction which becomes smooth instantly (i.e. for $t>0$ ) and satisfies (1.1).

Of course, the analysis of the weak flow as $t \rightarrow 0^{+}$is of great importance, and we have some discussion in Section 4 with geometric interests. 
Formally, we see $\left[\widetilde{\omega}_{t}\right]=\left[\omega_{t}\right] \in H^{1,1}(X, \mathbb{C}) \cap H^{2}(X, \mathbb{R})$, where

$$
\omega_{t}=\omega_{\infty}+e^{-t}\left(\omega-\omega_{\infty}\right)
$$

with $\omega_{\infty}=-\operatorname{Ric}(\Omega):=\sqrt{-1} \partial \bar{\partial} \log \left(\frac{\Omega}{(\sqrt{-1})^{n} d z_{1} \wedge d \bar{z}_{1} \wedge \cdots \wedge d z_{n} \wedge d \bar{z}_{n}}\right)$ in a local coordinate system $\left\{z_{1}, \cdots, z_{n}\right\}$. One has $\left[\omega_{\infty}\right]=-c_{1}(X)=K_{X}$. The Main Assumption above simply means

$$
\left[\omega_{t}\right]=e^{-t}[\omega]+\left(1-e^{-t}\right) K_{X}
$$

is Kähler for $t \in(0, T)$ for some $T>0$. It is now routine to see that at least formally (1.1) would be equivalent to the following evolution equation for a spacetime function $u$ :

$$
\frac{\partial u}{\partial t}=\log \frac{\left(\omega_{t}+\sqrt{-1} \partial \bar{\partial} u\right)^{n}}{\Omega}-u, u(\cdot, 0)=v,
$$

with the understanding of $\widetilde{\omega}_{t}=\omega_{t}+\sqrt{-1} \partial \bar{\partial} u$. Just as for the classic smooth KählerRicci flow, we'll focus on defining the weak version of (1.2) instead of (1.1). Their equivalence in the category of smooth objects would make the weak version for (1.2) naturally the weak version for (1.1) in light of the smoothing effect described in Theorem 1.2 .

Now it's time to describe the Approximation Construction involved in the statement of Theorem 1.2, which is similar to what has been applied in [3] except that now $[\omega]$ is no longer Kähler. The idea is to find approximation of the initial data, use them as initial data to get a sequence of flows, and finally take limit of the flows. We provide the details below for the reader's convenience.

Take some Kähler metric $\omega_{1}$ over $X$. For any $\epsilon \geqslant 0$, set $\omega(\epsilon)=\omega+\epsilon \omega_{1}$ and $\omega_{t}(\epsilon)=\omega_{\infty}+e^{-t}\left(\omega(\epsilon)-\omega_{\infty}\right)$. Using the regularization result in [2], one has for any sequence $\left\{\epsilon_{j}\right\}$ decreasing to 0 as $j \rightarrow \infty$, a sequence of functions $\left\{v_{j}\right\}$ with $v_{j} \in C^{\infty}(X)$ and $\omega\left(\epsilon_{j}\right)+\sqrt{-1} \partial \bar{\partial} v_{j}>0$, decreasing to $v$ accordingly. Then we consider the Kähler-Ricci flows,

$$
\frac{\partial \widetilde{\omega}_{t}\left(\epsilon_{j}\right)}{\partial t}=-\operatorname{Ric}\left(\widetilde{\omega}_{t}\left(\epsilon_{j}\right)\right)-\widetilde{\omega}_{t}\left(\epsilon_{j}\right), \widetilde{\omega}_{0}(\epsilon)=\omega\left(\epsilon_{j}\right)+\sqrt{-1} \partial \bar{\partial} v_{j} .
$$

At the level of metric potential, we have

$$
\frac{\partial u_{j}}{\partial t}=\log \frac{\left(\omega_{t}\left(\epsilon_{j}\right)+\sqrt{-1} \partial \bar{\partial} u_{j}\right)^{n}}{\Omega}-u_{j}, u_{j}(\cdot, 0)=v_{j} .
$$

They are the classic smooth Kähler-Ricci flows. By choosing the $T$ (before (1.2)) properly, all these flows for $j \gg 1$ (i.e. $\epsilon_{j}$ sufficiently small) would exist for $t \in[0, T)$ from cohomology consideration 1

In light of $v_{j}$ and $\omega_{t}\left(\epsilon_{j}\right)$ "decreasing" to $v$ and $\omega_{t}$ as $j \rightarrow \infty$, applying the Maximum Principle, one can see that $u_{j}$ is also decreasing as $j \rightarrow \infty$. In principle, this would allow us to get a limit for each $t \in[0, T), u(\cdot, t) \in P S H_{\omega_{t}}(X)$, which is the desired weak flow. For this to be true literally, one needs to make sure that for each such $t$, the decreasing limit of $u_{j}(\cdot, t)$ won't be $-\infty$ uniformly over $X$. At the initial time, this is obviously the case. For $t \in(0, T)$, this would be the case as seen later by applying Kolodziej's $L^{\infty}$-estimate (as in [10]) for Kähler classes 2

\footnotetext{
${ }^{1} \mathrm{As}$ in [3], it's the short time existence that's of interest, i.e. a small interval near $t=0$.

${ }^{2}$ One can also achieve this using the classic PDE argument involving Moser Iteration (see 19 ] for example).
} 
Of course, one then needs to make sure that the limit, which is at this moment just a family of positive $(1,1)$-current with parameter $t$, is more classic, and this would boil down to obtaining uniform estimates of $u_{j}$ for all $j$ 's.

Before heading into the search for those uniform estimates, let's justify the uniqueness statement of Theorem 1.2, which is a straightforward application of the Maximum Principle.

To begin with, let's observe that the decreasing convergence of $v_{j} \rightarrow v$ can be modified to strictly decreasing convergence without changing the limit. In fact, if $\left\{v_{j}\right\}$ is only a decreasing sequence, then $\left\{v_{j}+\frac{1}{j}\right\}$ is strictly decreasing with the same limit. Clearly, by the affect on the solution of (1.4), $u_{j}$ is negligible as $j \rightarrow \infty$. Also, the decreasing limit won't be affected by taking subsequence.

Now we can see that the choice of sequence $\left\{\epsilon_{j}\right\}$ won't affect the limit (i.e. the weak flow). Let's take two strictly decreasing sequences, $\left\{v_{j}\right\}$ and $\left\{v_{\alpha}\right\}$ in the construction described before (1.3). Since for each $j, v_{j}>v$ and $v_{\alpha}$ decreases to $v$, by Dini's Theorem (since all the functions involved are smooth), $v_{\alpha}<v_{j}$ for $\alpha$ sufficiently large. The other direction is similar. So by taking subsequences, denoted by $\left\{v_{j_{a}}\right\}$ and $\left\{v_{\alpha_{b}}\right\}$, we have

$$
v_{j_{1}}>v_{\alpha_{1}}>v_{j_{2}}>v_{\alpha_{2}}>\cdots .
$$

Applying the Maximum Principle to (1.4) (the difference of two, to be more precise), one has

$$
u_{j_{1}}>u_{\alpha_{1}}>u_{j_{2}}>u_{\alpha_{2}}>\cdots,
$$

and so they have the same limit.

Now one can take care of the general case. In the construction before (1.3), suppose we have chosen different $\omega_{1}$ 's with different strictly decreasing sequences $\left\{v_{j}\right\}$. Then we can recycle the argument above while also making sure the corresponding $\omega_{t}\left(\epsilon_{j}\right)$ 's have the same comparison relation. Thus the Maximum Principle would still give the same kind of comparison for solutions of (1.4). Hence the limit would still be the same and we can conclude the uniqueness.

Remark 1.3. By all means, using approximation is the only reasonable way to come up with a weak flow, and so even without any description of the situation as $t \rightarrow 0^{+}$, it is still quite natural to call this the weak flow initiating from the current $\omega_{0}=\omega+\sqrt{-1} \partial \bar{\partial} v$. The discussion for the situation as $t \rightarrow 0^{+}$is more for the backwards uniqueness of the weak flow. One can find discussion on backwards uniqueness for smooth Ricci flow in 12 .

\section{Estimates AWAY FROM INITIAL TIME}

Now we begin the search for estimates uniform for all approximation flows (i.e. $\epsilon>0$, where we have classic smooth flows). For simplicity, we'll omit $j$ and $\epsilon_{j}$ in the notation below, which would unfortunately make (1.3) and (1.4) look exactly like (1.1) and (1.2) respectively. However this would also help us keep in mind the degeneracy of the background form, and so is not such a terrible choice considering what we are trying to do here.

Note. $C$ below would stand for a fixed positive constant which might be different at places. Its dependence on other constants would be clear from the context. Moreover, $L^{p>1}$ stands for $L^{p}$ for some $p>1$, where the $p$ can also be different at places but is a fixed one at each place. 
Clearly, $u \leqslant C$ by the Maximum Principle for (1.4). This is uniform as long as the flow exists. For the other estimates, the idea is to try to eliminate the affect of the initial data as completely as possible, because we don't have control of the initial data except for the $L^{\infty}$-bound of the metric potential. Also recall that we only need estimates for small time.

Notice that in (1.4), the initial value of the background form, $\omega$, might not be non-negative. One can actually make better use of that $\omega+\sqrt{-1} \partial \bar{\partial} v>0$ by looking at the following evolution at the level of metric potential for the same flow (1.3),

$$
\frac{\partial \phi}{\partial t}=\log \frac{\left(\widehat{\omega}_{t}+\sqrt{-1} \partial \bar{\partial} \phi\right)^{n}}{\Omega}-\phi, \phi(\cdot, 0)=0,
$$

where $\widehat{\omega}_{t}=\omega_{\infty}+e^{-t}\left(\omega+\sqrt{-1} \partial \bar{\partial} v-\omega_{\infty}\right)$. It's easy to see the relation between the solutions of (1.4) and (2.1), which is $u=\phi+e^{-t} \cdot v$. We have $\phi \leqslant C$ from $u \leqslant C$, which is not clear by applying the Maximum Principle to (2.1) because of the lack of uniform control for $\omega+\sqrt{-1} \partial \bar{\partial} v$ as a form in approximation.

The following equation is obtained by taking the $t$-derivative of (2.1) and playing some standard transformations:

$$
\frac{\partial}{\partial t}\left(\left(e^{t}-1\right) \frac{\partial \phi}{\partial t}-\phi\right)=\Delta_{\widetilde{\omega}_{t}}\left(\left(e^{t}-1\right) \frac{\partial \phi}{\partial t}-\phi\right)+n-\left\langle\widetilde{\omega}_{t}, \omega+\sqrt{-1} \partial \bar{\partial} v\right\rangle .
$$

Since $\omega+\sqrt{-1} \partial \bar{\partial} v>0$, applying the Maximum Principle and noticing the lower bound of the initial value and the uniform upper bound of $\phi$, we have for $t \in(0, T)$,

$$
\frac{\partial \phi}{\partial t} \leqslant \frac{C}{e^{t}-1},
$$

which gives the following bound of $\frac{\partial u}{\partial t}$ since $\frac{\partial u}{\partial t}=\frac{\partial \phi}{\partial t}-e^{-t} \cdot v$ :

$$
\frac{\partial u}{\partial t} \leqslant \frac{C}{e^{t}-1} .
$$

For any $t \in(0, T)$, we have the background form $\left[\omega_{t}(\epsilon)\right]$ being uniformly Kähler, i.e. the small interval corresponding to $\epsilon$ is in the Kähler cone. Together with the above upper bound for $\frac{\partial u}{\partial t}$, one can apply Kołodziej's $L^{\infty}$-estimate (as in [10]) for (1.4) in the form of

$$
\left(\omega_{t}+\sqrt{-1} \partial \bar{\partial} u\right)^{n}=e^{\frac{\partial u}{\partial t}+u} \Omega
$$

to achieve the $L^{\infty}$-bound for $u$. So now we have $u(\cdot, t) \geqslant-C(t)$ with $C(t)$ finite for $t \in(0, T)$. In fact, we know by the result in [11] that $u(\cdot, t)$ is Hölder continuous for these $t$ 's 3

Remark 2.1. The original results on the $L^{\infty}$-estimate (as in [10], [20] and [21]) are usually stated for Monge-Ampère equations in the form $(\omega+\sqrt{-1} \partial \bar{\partial} u)^{n}=f \cdot \Omega$, where $[\omega]$ might be degenerate, $f \geqslant 0$ is in some $L^{p>1}$-space, and $\Omega$ is a (nondegenerate) smooth volume form. There is more than one way to translate this when applying it to the above equation with $e^{u}$ on the right hand side.

Method I: get the $L^{p}$-bound for the measure $f \cdot e^{u} \Omega$; then one knows the normalized $u$ would be bounded from the original result. In other words, $u$ only takes value in some interval with well-controlled length. Then the upper bound of $u$, which usually comes from the direct Maximum Principle argument, together with the upper bound for $f \cdot \Omega$ which guarantees $u$ cannot take too small a value in sight of the lower bound for the total volume, would provide the bound for $u$.

\footnotetext{
${ }^{3}$ The Hölder exponent will also depend on $t$.
} 
Method II: get the $L^{p}$-bound for the measure $f \cdot \Omega$; then consider the equation $(\omega+\sqrt{-1} \partial \bar{\partial} w)^{n}=C f \cdot \Omega$. The idea is to apply the Maximum Principle to the quotient of these two equations. In order to control the (normalized) solution $w$ for this auxiliary equation, one needs to control the constant $C$ (from above), which means we need a lower bound for the total volume for the measure $f \cdot \Omega$. Again this can be achieved from the upper bound for $u$.

It's not hard to see that these two methods are merely different combinations of the same set of information.

Since for any $t \in\left[\lambda_{1}, \lambda_{2}\right] \subset(0, T)$, [ $\left.\omega_{t}\right]$ is uniformly Kähler (for any approximation flow), by properly choosing $\Omega$ (and so $\omega_{\infty}$ ), one has $\omega_{t}$ being uniform as a Kähler metric for $t \in\left[\lambda_{1}, \lambda_{2}\right] 4$ Clearly, $(0, T)$ can be exhausted by such intervals (and we only care for the end at 0 ).

Now let's translate the time, making $\lambda_{1}$ the new initial time $t=0$. From the discussion before, we have uniform bounds from both sides for $u$ and the uniform upper bound for $\frac{\partial u}{\partial t}$. By taking the $t$-derivative for (1.4) and making some transformations, we have the two equations below:

$$
\begin{gathered}
\frac{\partial}{\partial t}\left(\left(e^{t}-1\right) \frac{\partial u}{\partial t}\right)=\Delta_{\widetilde{\omega}_{t}}\left(\left(e^{t}-1\right) \frac{\partial u}{\partial t}\right)-\left(1-e^{-t}\right)\left\langle\widetilde{\omega}_{t}, \omega-\omega_{\infty}\right\rangle+\frac{\partial u}{\partial t} \\
\frac{\partial}{\partial t}\left(\left(e^{t}-1\right) \frac{\partial u}{\partial t}-u\right)=\Delta_{\widetilde{\omega}_{t}}\left(\left(e^{t}-1\right) \frac{\partial u}{\partial t}-u\right)+n-\left\langle\widetilde{\omega}_{t}, \omega\right\rangle .
\end{gathered}
$$

Notice that the $\omega$ here is indeed $\omega_{\lambda_{1}}$ and is uniform as a Kähler metric for all approximation flows.

In the small time interval $\left[0, \lambda_{2}-\lambda_{1}\right]$ (after translation), we have made sure that $\omega_{t}>0$, which is

$$
\omega_{\infty}+e^{-t}\left(\omega-\omega_{\infty}\right)=\omega-\left(1-e^{-t}\right)\left(\omega-\omega_{\infty}\right)>0,
$$

so one can choose $\delta \in(0,1)$ such that for such time,

$$
\delta \omega-\left(1-e^{-t}\right)\left(\omega-\omega_{\infty}\right)>0 .
$$

Use this $\delta$ to multiply (2.3) and take the difference with (2.2) to arrive at

$$
\begin{aligned}
\frac{\partial}{\partial t}\left((1-\delta)\left(e^{t}-1\right) \frac{\partial u}{\partial t}+\delta u\right)= & \Delta_{\widetilde{\omega}_{t}}\left((1-\delta)\left(e^{t}-1\right) \frac{\partial u}{\partial t}+\delta u\right) \\
& +\left\langle\widetilde{\omega}_{t}, \delta \omega-\left(1-e^{-t}\right)\left(\omega-\omega_{\infty}\right)\right\rangle+\frac{\partial u}{\partial t}-n \delta .
\end{aligned}
$$

Consider the (local in time) minimal value point of $(1-\delta)\left(e^{t}-1\right) \frac{\partial u}{\partial t}+\delta u$, which would be bounded if it's taken at the initial time. If it is not at the (new) initial time, then at that point, we have

$$
\begin{aligned}
\left\langle\widetilde{\omega}_{t}, \delta \omega-\left(1-e^{-t}\right)\left(\omega-\omega_{\infty}\right)\right\rangle & \geqslant n \cdot\left(\frac{\left(\delta \omega-\left(1-e^{-t}\right)\left(\omega-\omega_{\infty}\right)\right)^{n}}{\widetilde{\omega}_{t}^{n}}\right)^{\frac{1}{n}} \\
& =n \cdot\left(\frac{\left(\delta \omega-\left(1-e^{-t}\right)\left(\omega-\omega_{\infty}\right)\right)^{n}}{e^{\frac{\partial u}{\partial t}+u} \Omega}\right)^{\frac{1}{n}} \\
& \geqslant C e^{-\frac{1}{n} \frac{\partial u}{\partial t}},
\end{aligned}
$$

\footnotetext{
${ }^{4}$ In fact, one only needs to take care of the case $\epsilon=0$ for this. It's merely going to cause a difference for the evolution equations at the level of metric potential in a way similar to that between (1.4) and (2.1).
} 
where $u \leqslant C$ is applied in the last step, and so one concludes

$$
C \geqslant C e^{-\frac{\partial u}{\partial t}}+\frac{\partial u}{\partial t}
$$

which gives $\frac{\partial u}{\partial t} \geqslant-C$ at that point. Thus $(1-\delta)\left(e^{t}-1\right) \frac{\partial u}{\partial t}+\delta u \geqslant-C$ at that point by the known lower bound of $u$, and so we conclude

$$
(1-\delta)\left(e^{t}-1\right) \frac{\partial u}{\partial t}+\delta u \geqslant-C
$$

for the space-time. In sight of the upper bound for $u$, we arrive at

$$
\frac{\partial u}{\partial t} \geqslant-\frac{C}{e^{t}-1} \text {. }
$$

Although this time $t$ has been translated in the above argument, one still achieves the lower bound for $\frac{\partial u}{\partial t}$ uniformly for all approximation flows away from the original initial time.

So far, we have obtained the $L^{\infty}$-bounds for both $u$ and $\frac{\partial u}{\partial t}$ locally away from the initial time. Only the upper bound of $u$ is uniform for all time.

The second and higher order estimates can be carried through as in Subsection 3.2 of [3] because the translation of time would make the background form Kähler. Hence we conclude that the weak flow defined in Section 1 becomes smooth instantly. The proof of Theorem 1.2 is thus finished.

Remark 2.2. The situation as $t \rightarrow 0^{+}$, which is indeed the only "weak" spot of the flow, needs further consideration just as in [3. Since most estimates achieved up to this point are only local away from the initial time, the control of the situation near 0 at this moment is very weak. In fact, strange things can happen for the weak flow. For example, $\left[\omega_{0}\right]$ might have global volume 0 (being collapsed), but the volume would become positive instantly.

\section{Revisiting the KÄHLER CASE}

In this section, we digress a little to the Kähler case, i.e. $[\omega]$ being Kähler, to sort out a very natural question regarding the uniqueness of weak flow from approximation construction. Recall that in [3], the approximation construction makes use of a fixed background form which is Kähler. There was no good reason at that time to use $\omega(\epsilon)=\omega+\epsilon \omega_{1}$ which appears in Section 1 here. However, the construction in Section 1 still works for that case.

Our goal here is to see that these two approximation constructions give rise to the same weak flow when $v$ is continuous. In the Kähler case, the continuity of $v$ follows from $(\omega+\sqrt{-1} \partial \bar{\partial} v)^{n}$ being $L^{p>1}$ by the result in [9], and so is a fairly reasonable assumption.

In [3], a sequence $\left\{\phi_{j}\right\}$ decreasing to $v$ for $\phi_{j} \in C^{\infty} \cap P S H_{\omega}(X)$ with $\omega+$ $\sqrt{-1} \partial \bar{\partial} \phi_{j}$ is used for approximation. The continuity of $v$ implies that the decreasing convergence is uniform by Dini's Theorem. The background form is the same for all $j$, and so by the Maximum Principle, the corresponding metric potentials for the approximation flows also uniformly converge to the weak flow with a continuous metric potential, called $\varphi$. This is the observation made in 15] which is crucial.

For the construction in Section 1, the continuity of $v$ also makes sure that the decreasing convergence of $v_{j}$ to $v$ used in Section 1 is uniform, but because the background form changes for this approximation, we still only know that the convergence of $u_{j}$ is decreasing, with the weak flow $u$. 
In fact, applying the Maximum Principle between these two approximations, one easily has $u \geqslant \varphi$, but the equality is what we want.

To begin with, we prove that $u$ is continuous up to the initial time. By the discussion in Section 2, we only need to check at the initial time, where $u(\cdot, 0)=v$. The decreasing convergence of $u_{j}$ to $u$ makes sure that $u$ is upper semi-continuous. For any $\epsilon>0$ and $p \in X$, we have $\delta_{1}>0$ such that for $(x, t)$ with $\operatorname{dist}_{\omega_{1}}(x, p)+t<\delta_{1}$ (where $\omega_{1}$ is a fixed metric over $X$ ),

$$
u(x, t) \leqslant u(p, 0)+\epsilon=v(p)+\epsilon .
$$

We have $\varphi \in C^{0}(X \times[0, T))$, and so there is $\delta_{2}>0$ such that for $(x, t)$ with $\operatorname{dist}_{\omega_{1}}(x, p)+t<\delta_{2}$,

$$
\varphi(x, t) \geqslant \varphi(0, p)-\epsilon=v(p)-\epsilon .
$$

Let $\delta=\min \left\{\delta_{1}, \delta_{2}\right\}$. We have for $(x, t) \in U(p)=\left\{(x, t) \mid \operatorname{dist}_{\omega_{1}}(x, p)+t<\delta\right\}$,

$$
u(x, t) \leqslant \varphi(x, t)+2 \epsilon .
$$

Finitely many $U(p)$ 's would cover $X \times\{0\}$, and so for some $\delta(\epsilon)>0$,

$$
u(x, t) \leqslant \varphi(x, t)+2 \epsilon, \text { for any }(x, t) \in X \times[0, \delta(\epsilon)] .
$$

Combining with $u \geqslant \varphi$, one can easily conclude the continuity for $u$ at the initial time. So we have $u \in C^{0}(X \times[0, T))$.

From [3] and Section 2 here, we also know $u$ and $\varphi$ are both in $C^{\infty}(X \times(0, T))$ and satisfying

$$
\frac{\partial u}{\partial t}=\log \frac{\left(\omega_{t}+\sqrt{-1} \partial \bar{\partial} u\right)^{n}}{\Omega}-u, \frac{\partial \varphi}{\partial t}=\log \frac{\left(\omega_{t}+\sqrt{-1} \partial \bar{\partial} \varphi\right)^{n}}{\Omega}-\varphi
$$

with the same background form $\omega_{t}$. Moreover, they are both in $C^{0}(X \times[0, T))$ with $u(\cdot, 0)=\varphi(\cdot, 0)=v(\cdot)$. So for any $\epsilon>0$, we can have for sufficiently small $\delta>0,|u(\cdot, \delta)-\varphi(\cdot, \delta)| \leqslant \epsilon$. Applying the Maximum Principle for

$$
\frac{\partial(u-\varphi)}{\partial t}=\log \frac{\left(\omega_{t}+\sqrt{-1} \partial \bar{\partial} \varphi+\sqrt{-1} \partial \bar{\partial}(u-\varphi)\right)^{n}}{\left(\omega_{t}+\sqrt{-1} \partial \bar{\partial} \varphi\right)^{n}}-(u-\varphi)
$$

over $X \times[\delta, T)$, we have $|u-\varphi| \leqslant \epsilon$ over this region. Hence $u=\varphi$ and we end up with the same weak flow.

\section{Estimates up to initial time}

In this part, we look to achieve some estimates uniform for small time, i.e. for $t \in(0, T)$ without degeneration towards $t=0$. So far, we only have the uniform upper bound for $u$.

The first thing that comes to mind could be some $L^{\infty}$-estimate for the metric potential, $u$, up to $t=0$. In sight of the Kołodziej type of estimates (in [7, 21] and [5]) and the even more elementary relation between $\frac{\partial u}{\partial t}$ and $u$, one naturally wants to control the volume form or $\frac{\partial u}{\partial t}$ up to the initial time. Although one might think that some bound of $\frac{\partial u}{\partial t}$ might already control $u$ (to some extent) without the involvement of the Kolodziej type of estimates for the degenerate case because of the uniform control for $u$ at some positive time from Section 2, we still need them when constructing the approximation flows as discussed below.

As in [3], we often make an assumption on the initial measure, $\omega_{0}^{n}=(\omega+$ $\sqrt{-1} \partial \bar{\partial} v)^{n}$, which is closely related to the initial value of $\frac{\partial u}{\partial t}$. However, if one just applies the general regularization of the current $\omega_{0}$ (in [2 for example), it may 
not be the case that the volume forms of the smooth approximation metrics would maintain the same kind of control for their Monge-Ampère measures. Fortunately, the uniqueness of the weak flow in Theorem 1.2 allows us to make a good choice of the approximation, maintaining the measure control. More precisely, suppose the measure $(\omega+\sqrt{-1} \partial \bar{\partial} v)^{n}$ has some kind of bound (for example, upper, lower or $L^{p>1}$-bounds, even degenerate ones). Then one can use the standard process involving partition of unity and convolution to construct a sequence of smooth volume forms, $\Omega_{\epsilon}$, with the same kind of bound uniformly and which converges to $(\omega+\sqrt{-1} \partial \bar{\partial} v)^{n}$ as $\epsilon \rightarrow 0$ in $L^{1}$-space. Then we need to solve the equation $\left(\omega+\epsilon \omega_{1}+\sqrt{-1} \partial \bar{\partial} v_{\epsilon}\right)^{n}=C_{\epsilon} \Omega_{\epsilon}$ where $C_{\epsilon}$ is well controlled and tends to 1 by requiring $\int_{X}(\omega+\sqrt{-1} \partial \bar{\partial} v)^{n}>0$. We also need to make sure that after taking normalization for $v_{\epsilon}$, they would decrease to $v$ as $\epsilon \rightarrow 0$, so that they form a desirable approximation in the construction of the weak flow.

For the existence of such $v_{\epsilon}$, one needs the Kołodziej type of result (as in [7, 21] and [5]), and so we assume the degenerate initial class $\left[\omega_{0}\right]$ to be large as in [7] and [5], or in the following more intuitive but seemingly more restrictive picture: let $X$ be a closed Kähler manifold with $\operatorname{dim}_{\mathbb{C}} X=n \geqslant 2$, and there is a holomorphic map $P: X \rightarrow \mathbb{C P}^{N}$ with $\operatorname{dim}_{\mathbb{C}} F(X)=n$. Let $\omega_{M}$ be any (smooth) Kähler form over some neighborhood of $P(X)$ in $\mathbb{C P}^{N},\left[\omega_{0}\right]=\left[P^{*} \omega_{M}\right]$. Moreover, we also need the measure $(\omega+\sqrt{-1} \partial \bar{\partial} v)^{n}$ to be $L^{p>1}$ (or slightly more general as in [5]). The decreasing of $v_{\epsilon}$ is clear using Kołodziej's stability argument as discussed in 9.6.2 of 22 . The stability result in [6] then guarantees that the limit of $v_{\epsilon}$ is $v$.

With all the above observations, we analyze the situation as $t \rightarrow 0^{+}$for some cases of interest. We are still working directly on the approximation flows, i.e. (1.3) and (1.4), while omitting $j$ and $\epsilon_{j}$ for simplicity of notation. We emphasize that the argument is uniform for all approximation flows.

4.1. Measure of the upper bound. We search for the upper bound of $\frac{\partial u}{\partial t}$ uniform up to the initial time under the assumption that the volume of the initial current, $\omega_{0}^{n}$, has some appropriate upper bound. The point is to see whether it would be enough to imply the necessary measure bound for a small time interval. In the following, we would provide enough motivation before stating the actual bound (4.4).

The following equation comes from standard manipulation of (1.4):

$$
\frac{\partial}{\partial t}\left(\left(e^{t}-A\right) \frac{\partial u}{\partial t}-A u\right)=\Delta_{\widetilde{\omega}_{t}}\left(\left(e^{t}-A\right) \frac{\partial u}{\partial t}-A u\right)+A n-\left\langle\widetilde{\omega}_{t}, \omega-(1-A) \omega_{\infty}\right\rangle
$$

for a constant $A$ to be fixed. Since the flow is driving the class into the Kähler cone, it would take some $A>1$ to provide a definite sign for the last term on the right hand side of this equation. Unfortunately, when applying the Maximum Principle, we would not have the proper control for the initial value of $(1-A) \frac{\partial u}{\partial t}$ (from the upper bound for $\frac{\partial u}{\partial t}$ at the initial time).

So one needs to choose some constant $A<1$. It would work if there is an effective divisor (i.e. a holomorphic line bundle) $E$ with the defining section $\sigma$ and a hermitian metric $|\cdot|$ such that for some $\lambda>0$,

$$
\omega+\lambda \sqrt{-1} \partial \bar{\partial} \log |\sigma|^{2}>0,
$$

which is the case under our assumption on $\left[\omega_{0}\right]$ in the algebraic geometry setting, i.e. $\left[\omega_{0}\right]-\lambda E$ being Kähler. This can be justified using the simple algebraic 
geometry result as cited in 20. We would assume this in general, which is indeed an assumption on the geometry of the (effective and Kähler) cones. This would be made clear in the statement of the result. Clearly, we can also make sure that $|\sigma|$ is small over $X$. Now one can reformulate (4.1) as follows:

$$
\begin{aligned}
\frac{\partial}{\partial t}\left(\left(e^{t}-A\right) \frac{\partial u}{\partial t}-A u+\lambda \log |\sigma|^{2}\right) & =\Delta_{\widetilde{\omega}_{t}}\left(\left(e^{t}-A\right) \frac{\partial u}{\partial t}-A u+\lambda \log |\sigma|^{2}\right)+A n \\
& -\left\langle\widetilde{\omega}_{t}, \omega+\lambda \sqrt{-1} \partial \bar{\partial} \log |\sigma|^{2}-(1-A) \omega_{\infty}\right\rangle .
\end{aligned}
$$

By choosing $A<1$ properly, one can make sure that the last term on the right has a definite sign for the small time interval.

Recall that in this part we assume $\left.\frac{\partial u}{\partial t}\right|_{t=0} \leqslant C$; in other words, $\omega_{0}^{n} \leqslant C \cdot \Omega$. The discussion at the beginning of this section guarantees that we also have this for the approximation flows worked on directly. The Maximum Principle gives

$$
\left(e^{t}-A\right) \frac{\partial u}{\partial t}-A u+\lambda \log |\sigma|^{2} \leqslant \max _{X \times\{0\}}(1-A) \frac{\partial u}{\partial t}+\lambda \log |\sigma|^{2}+C \leqslant C,
$$

using the upper bound for $\frac{\partial u}{\partial t}$ and lower bound for $u$, both at the initial time. With the uniform upper bound for $u$ and $A<1$, one arrives at the following degenerate upper bound:

$$
\frac{\partial u}{\partial t} \leqslant \frac{-\lambda \log |\sigma|^{2}+C}{e^{t}-A}
$$

This degenerate upper bound (4.3) gets better for large $t$. Obviously, the assumption on the initial value can be weakened to

$$
\left.\frac{\partial u}{\partial t}\right|_{t=0} \leqslant \frac{-\lambda \log |\sigma|^{2}+C}{1-A},
$$

and of course we still require $\omega_{0}^{n}$ to be $L^{p>1}$, which is preserved by the approximation flows as discussed before. (4.3) is uniform for the approximation flows, and so is true for the weak flow for $t>0$ by the local smooth convergence in Section 2. Hence we conclude the following result.

Proposition 4.1. In the setting of Theorem 1.2, if $\omega_{0}$ has $L^{p>1}$-measure satisfying (4.4) and represents a big class, then the weak flow defined in Theorem 1.2 would satisfy (4.3).

The degenerate upper bound of $\frac{\partial u}{\partial t}$, namely (4.3), means that locally out of $\{\sigma=0\} 5 u$ is decreasing up to a term like $-C t$ as $t \nearrow$. More precisely, $u+C \log |\sigma|^{2} t$ is decreasing as $t \nearrow$.

Using the notation in Section 1, because this is uniform for the approximation flow $u_{j}$, we have that $u_{j}+C \log |\sigma|^{2} t$ increases to $v_{j}$ as $t \searrow 0$, while $v_{j}$ decreases to $v$ and $u_{j}$ decreases to $u$ as $j \rightarrow \infty$. Notice that we also know that for the genuine weak flow, $u+C \log |\sigma|^{2} t$ increases as $t \searrow 0$ because of the smooth convergence away

\footnotetext{
${ }^{5}$ This can be improved to be the stable base locus set of $[\omega]$.
} 
from the initial time in Section 2. So we can have the following over $X \backslash\{\sigma=0\}$ :

$$
\begin{aligned}
v(\cdot) & =\lim _{j \rightarrow \infty} v_{j}(\cdot)=\inf _{j} v_{j}(\cdot) \\
& =\inf _{j} \sup _{t}\left\{u_{j}(\cdot, t)+C \log |\sigma|^{2} t\right\} \\
& \geqslant \sup _{t} \inf _{j}\left\{u_{j}(\cdot, t)+C \log |\sigma|^{2} t\right\} \\
& =\sup _{t}\left\{u(\cdot, t)+C \log |\sigma|^{2} t\right\} \\
& =\lim _{t \rightarrow 0^{+}}\left(u(\cdot, t)+C \log |\sigma|^{2} t\right)=\lim _{t \rightarrow 0^{+}} u(\cdot, t) .
\end{aligned}
$$

This leads to a key issue for the weak flow as $t \rightarrow 0^{+}$as discussed below. We define $\hat{v}(\cdot)=\lim _{t \rightarrow 0^{+}} u(\cdot, t)$ out of $\{\sigma=0\}$. Locally out of $\{\sigma=0\}$, there is certainly a lower bound for $\hat{v}$ because of the increasing of $u(\cdot, t)+C \log |\sigma|^{2} t$ as $t \searrow 0$ and the $L^{\infty}$-bound for $u$ away from the initial time. The uniform upper bound is also available. So we can extend $\hat{v}$ to $\{\sigma=0\}$ in an upper semi-continuous way so that $\hat{v} \in P S H_{\omega}(X)$ (as in 4 for instance). Moreover, we have

$$
C \geqslant \hat{v} \geqslant-C_{\epsilon}+\epsilon \log |\sigma|^{2}
$$

for any (small) $\epsilon>0$. In general, we have $v \geqslant \hat{v}$. The equality is expected.

Set $\widehat{\omega}_{0}=\omega+\sqrt{-1} \partial \bar{\partial} \hat{v}$. By the classic result on weak convergence (in 1] or as summarized in [10]), for the weak flow,

$$
\widetilde{\omega}_{t}^{j} \rightarrow \widehat{\omega}_{0}^{j} \text { weakly over } X \backslash\{\sigma=0\} \text { as } t \rightarrow 0^{+}, j=1, \cdots, n .
$$

In fact, one can conclude the weak convergence over $X$ for the Monge-Ampère measure with the additional assumption that $\hat{v} \in L^{\infty}(X) 6$ This would be the case if the positive quantity $\frac{\lambda}{1-A}$ in (4.3) could be small enough, because the uniform $L^{p>1}$-bound for the measure $e^{\frac{\partial u}{\partial t}} \Omega$ would then be available in light of (4.4). This leads to the uniform $L^{\infty}$-bound for the metric potential $u_{j}$ for the approximation flows by the Kołodziej type of $L^{\infty}$-estimate.

To see the weak convergence over $X$, select a sequence of strictly increasing sets exhausting $X \backslash\{\sigma=0\},\left\{U_{k}\right\}$ with non-negative smooth functions $\left\{\rho_{k}\right\}$ supported on $U_{k+1}$ and equal to 1 over $U_{k}$. Because of the obvious convergence from the global cohomology data,

$$
\int_{X} \widetilde{\omega}_{t}^{j} \cdot \omega_{1}^{n-j} \rightarrow \int_{X} \widehat{\omega}_{0}^{j} \cdot \omega_{1}^{n-j} \text { as } t \rightarrow 0^{+}, j=1, \cdots, n,
$$

for any Kähler metric $\omega_{1}$, one only needs to consider non-negative $(n-j, n-j)$-forms as the test form. For any one of those, called $G$ over $X$, we have

$$
\lim _{t \rightarrow 0^{+}} \int_{X} \rho_{k} \cdot G \cdot \widetilde{\omega}_{t}^{j}=\int_{X} \rho_{k} \cdot G \cdot \widehat{\omega}_{0}^{j}
$$

from the local weak convergence over $X \backslash\{\sigma=0\}$. We then take supremum of the above convergence to get

$$
\sup _{k}\left(\lim _{t \rightarrow 0^{+}} \int_{X} \rho_{k} \cdot G \cdot \widetilde{\omega}_{t}^{j}\right)=\sup _{k}\left(\int_{X} \rho_{k} \cdot G \cdot \widehat{\omega}_{0}^{j}\right)=\int_{X} G \cdot \widehat{\omega}_{0}^{j} .
$$

\footnotetext{
${ }^{6}$ This is not so surprising as $\{\sigma=0\}$ is a pluripolar set.
} 
Meanwhile, we also have

$$
\begin{aligned}
\underline{\lim }_{t \rightarrow 0^{+}} \int_{X} G \cdot \widetilde{\omega}_{t}^{j} & =\underline{\lim }_{t \rightarrow 0^{+}} \sup _{k}\left(\int_{X} \rho_{k} \cdot G \cdot \widetilde{\omega}_{t}^{j}\right) \\
& \geqslant \sup _{k}\left(\lim _{t \rightarrow 0^{+}} \int_{X} \rho_{k} \cdot G \cdot \widetilde{\omega}_{t}^{j}\right),
\end{aligned}
$$

so we arrive at

$$
\varliminf_{t \rightarrow 0^{+}} \int_{X} G \cdot \widetilde{\omega}_{t}^{j} \geqslant \int_{X} G \cdot \widehat{\omega}_{0}^{j} .
$$

The equality has to hold for $G$ being a proper (wedge) power of any Kähler metric (from cohomology consideration), and so it is also true for any test form $G$. It is easier for $\varlimsup_{t \rightarrow 0^{+}}$since $\overline{\lim }_{t \rightarrow 0^{+}} \geqslant \underline{\lim }_{t \rightarrow 0^{+}}$. Finally, we conclude

$$
\widetilde{\omega}_{t}^{j} \rightarrow \widehat{\omega}_{0}^{j} \text { weakly over } X \text { as } t \rightarrow 0^{+}, j=1, \cdots, n \text {. }
$$

Remark 4.2. This weak convergence for all wedge powers is in principle stronger than merely the weak convergence of the metric itself, i.e. $j=1$. Meanwhile, for $j=1$, the weak convergence over $X$ is true without requiring $\hat{v}$ to be bounded, because the bound (4.6) for $\hat{v}$ guarantees $\widehat{\omega}_{0}$ would not charge the set $\{\sigma=0\}$ (see a similar discussion in Section 8 of [13]).

4.2. Measure of the lower bound. The consideration on the lower bound of flow metric measure for small time might seem a little bit strange at first, but it gets what we want in a more direct way. There is also an interesting application described at the end.

Suppose the initial measure has a positive lower bound, i.e.

$$
\left.\frac{\partial u}{\partial t}\right|_{t=0} \geqslant-C .
$$

This lower bound can be preserved for the approximation as discussed at the beginning of this section. Let's recall equation (4.2) which appeared before:

$$
\frac{\partial}{\partial t}\left(\left(e^{t}-A\right) \frac{\partial u}{\partial t}-A u\right)=\Delta_{\widetilde{\omega}_{t}}\left(\left(e^{t}-A\right) \frac{\partial u}{\partial t}-A u\right)+A n-\left\langle\widetilde{\omega}_{t}, \omega-(1-A) \omega_{\infty}\right\rangle .
$$

Now one chooses a proper constant $A>1$ so that the last term

$$
\left\langle\widetilde{\omega}_{t}, \omega-(1-A) \omega_{\infty}\right\rangle>0
$$

which can be done from the cohomology picture, after choosing $\omega_{\infty}$ (i.e. $\Omega$ ) properly. The Maximum Principle then gives

$$
\left(e^{t}-A\right) \frac{\partial u}{\partial t}-A u \leqslant \max _{X \times\{0\}}(1-A) \frac{\partial u}{\partial t}+C \leqslant C
$$

in light of the lower bound of $\frac{\partial u}{\partial t}$ at the initial time. Using the upper bound for $u$, we arrive at

$$
\left(e^{t}-A\right) \frac{\partial u}{\partial t} \leqslant C .
$$

Hence for small time such that $e^{t}-A \leqslant-C<0$, we have

$$
\frac{\partial u}{\partial t} \geqslant \frac{C}{e^{t}-A} \geqslant-C .
$$

This automatically gives a lower bound of $u$ for small time, and also the weak convergence of $\widetilde{\omega}_{t}^{j} \rightarrow \omega_{0}^{j}$ over $X$ as $t \rightarrow 0^{+}$for $j=1, \cdots, n$ from the monotonicity 
of $u+C t$, using the classic result in [1. Here we do not have the inequality as in (4.5) because there is now only "inf" and no "sup" is involved.

Proposition 4.3. In the setting of Theorem 1.2, if $\omega_{0}$ has $L^{p>1}$-measure with a positive lower bound and represents a big class, then the metric potential is uniformly bounded for a small time interval and

$$
\widetilde{\omega}_{t}^{j} \rightarrow \omega_{0}^{j} \text { weakly over } X \text { as } t \rightarrow 0^{+}, j=1, \cdots, n .
$$

One can also come up with the degenerate version just as in Subsection 4.1 using a weaker assumption $\left.\frac{\partial u}{\partial t}\right|_{t=0} \geqslant \frac{\epsilon \log |\sigma|^{2}}{A-1}-C$, i.e. $(1-A) \frac{\partial u}{\partial t}+\epsilon \log |\sigma|^{2} \leqslant C$ at the initial time and making sure that the Kähler form $\omega-(1-A) \omega_{\infty}$ dominates the additional $\epsilon \sqrt{-1} \partial \bar{\partial} \log |\sigma|^{2}$. However we prefer the version in the proposition because the situation actually occurs very naturally in the following example.

Example 4.4. Consider the classic smooth Kähler-Ricci flow. In the finite time singularity case, in sight of the semi-ample results in 8 , we can easily have examples when the singular class $\left[\omega_{T}\right]$ is semi-ample and big. By the Parabolic Schwarz Lemma (as in [14]), we have $\left\langle\widetilde{\omega}_{t}, \omega_{T}\right\rangle \leqslant C$, and so $\widetilde{\omega}_{t}^{n} \geqslant C \omega_{T}^{n}$. Here the semi-ample $\left[\omega_{T}\right]$ generates a map $P: X \rightarrow \mathbb{C P}^{N}$, and up to a multiple, $\omega_{T}=P^{*} \omega_{F S}$ with the standard Fubini-Study metric $\omega_{F S}$. $\left[\omega_{T}\right]$ being big means $P(X)$ is of the same complex dimension as $X$. If $P(X)$ is smooth, then the push-forward of $\widetilde{\omega}_{T}$ would be in the setting of Proposition 4.3. and so the weak flow over $P(X)$ would weakly converge back to the push-forward current.

This shows that sometimes we do have singular metrics with volume forms bounded away from 0 coming up naturally. It already makes the picture on the global weak flow over complex surfaces of general type quite satisfying, with weak convergences (for all wedge powers) from both sides of the singularity time.

\section{Final REMARKS}

We now state the following conjecture on the situation as $t \rightarrow 0^{+}$for the weak flow in the general setting of Section 1.

Definition 5.1. In the setting of Theorem 1.2, as $t \rightarrow 0^{+}$, one always has the following convergences in the weak sense:

$$
u(\cdot, t) \rightarrow v(\cdot), \widetilde{\omega}_{t}^{j} \rightarrow \omega_{0}^{j}, j=1, \cdots, n .
$$

Besides the importance of carrying out Tian's Program, this topic of analytic flavour naturally requires the combination of techniques and ideas from differential geometry and pluripotential theory, as indicated from the discussion in this note.

Remark 5.2. The recent work by J. Song and G. Tian, 15, considers the weak Kähler-Ricci flow with more involvement of algebraic geometry background, and the results are of different flavours. Simply speaking, the initial positive current is only of bounded potential in Sections 1 and 2. We would also like to point out that the more recent work by J. Song and B. Weinkove, [16], has achieved GromovHausdorff convergence for the example described above, certainly providing more geometric intuition for the picture. 


\section{ACKNOWLEDGMENT}

The author would like to thank Gang Tian for introducing him to this wonderful field and all the people who have supported this work. The collaboration on an earlier work with Xiuxiong Chen and Gang Tian has provided precious knowledge on this topic and valuable experience. Sławomir Dinew, Jian Song and other people's interest and suggestions were also important. Finally, one cannot say enough aboout the friendly environment of the mathematics departments at the University of Michigan at Ann Arbor and Sydney University, where this work was carried out.

\section{REFERENCES}

[1] Eric Bedford and B. A. Taylor, A new capacity for plurisubharmonic functions, Acta Math. 149 (1982), no. 1-2, 1-40, DOI 10.1007/BF02392348. MR674165 (84d:32024)

[2] Zbigniew Błocki and Sławomir Kołodziej, On regularization of plurisubharmonic functions on manifolds, Proc. Amer. Math. Soc. 135 (2007), no. 7, 2089-2093 (electronic), DOI 10.1090/S0002-9939-07-08858-2. MR2299485 (2008a:32029)

[3] X. X. Chen, G. Tian, and Z. Zhang, On the weak Kähler-Ricci flow, Trans. Amer. Math. Soc. 363 (2011), no. 6, 2849-2863, DOI 10.1090/S0002-9947-2011-05015-4. MR.2775789 (2012f:53136)

[4] Jean-Pierre Demailly, Complex analytic and algebraic geometry. Online book: agbook.ps.gz.

[5] Jean-Pierre Demailly and Nefton Pali, Degenerate complex Monge-Ampère equations over compact Kähler manifolds, Internat. J. Math. 21 (2010), no. 3, 357-405, DOI 10.1142/S0129167X10006070. MR2647006 (2012e:32039)

[6] Sławomir Dinew and Zhou Zhang, On stability and continuity of bounded solutions of degenerate complex Monge-Ampère equations over compact Kähler manifolds, Adv. Math. 225 (2010), no. 1, 367-388, DOI 10.1016/j.aim.2010.03.001. MR.2669357 (2011m:32073)

[7] Philippe Eyssidieux, Vincent Guedj, and Ahmed Zeriahi, Singular Kähler-Einstein metrics, J. Amer. Math. Soc. 22 (2009), no. 3, 607-639, DOI 10.1090/S0894-0347-09-00629-8. MR2505296(2010k:32031)

[8] Yujiro Kawamata, The cone of curves of algebraic varieties, Ann. of Math. (2) 119 (1984), no. 3, 603-633, DOI 10.2307/2007087. MR744865 (86c:14013b)

[9] Sławomir Kołodziej, The complex Monge-Ampère equation, Acta Math. 180 (1998), no. 1, 69-117, DOI 10.1007/BF02392879. MR1618325 (99h:32017)

[10] Sławomir Kołodziej, The complex Monge-Ampère equation and pluripotential theory, Mem. Amer. Math. Soc. 178 (2005), no. 840, x+64. MR2172891 (2006d:32054)

[11] Sławomir Kołodziej, Hölder continuity of solutions to the complex Monge-Ampère equation with the right-hand side in $L^{p}$ : the case of compact Kähler manifolds, Math. Ann. 342 (2008), no. 2, 379-386, DOI 10.1007/s00208-008-0239-y. MR2425147 (2009g:32079)

[12] Brett L. Kotschwar, Backwards uniqueness for the Ricci flow, Int. Math. Res. Not. IMRN 21 (2010), 4064-4097, DOI 10.1093/imrn/rnq022. MR2738351 (2012c:53100)

[13] John Lott and Zhou Zhang, Ricci flow on quasi-projective manifolds, Duke Math. J. 156 (2011), no. 1, 87-123, DOI 10.1215/00127094-2010-067. MR2746389 (2012c:53101)

[14] Jian Song and Gang Tian, The Kähler-Ricci flow on surfaces of positive Kodaira dimension, Invent. Math. 170 (2007), no. 3, 609-653, DOI 10.1007/s00222-007-0076-8. MR2357504 (2008m:32044)

[15] Jian Song and Gang Tian, The Kähler-Ricci flow through singularities. ArXiv:0909.4898 (math.DG).

[16] Jian Song and Ben Weinkove, Contracting exceptional divisors by the Kähler-Ricci flow. Duke Math. J. 162 (2013), no. 2, 367-415. MR3018957

[17] Gang Tian, New results and problems on Kähler-Ricci flow (English, with English and French summaries), Astérisque 322 (2008), 71-92. Géométrie différentielle, physique mathématique, mathématiques et société. II. MR2521654 (2011a:53131)

[18] Gang Tian, Geometry and nonlinear analysis, (Beijing, 2002), Higher Ed. Press, Beijing, 2002, pp. 475-493. MR.1989199 (2004j:53052) 
[19] Gang Tian, Kähler-Einstein metrics on algebraic manifolds, Transcendental methods in algebraic geometry (Cetraro, 1994), Lecture Notes in Math., vol. 1646, Springer, Berlin, 1996, pp. 143-185, DOI 10.1007/BFb0094304. MR1603624 (98j:32035)

[20] Gang Tian and Zhou Zhang, On the Kähler-Ricci flow on projective manifolds of general type, Chinese Ann. Math. Ser. B 27 (2006), no. 2, 179-192, DOI 10.1007/s11401-005-0533-x. MR.2243679 (2007c:32029)

[21] Zhou Zhang, On degenerate Monge-Ampère equations over closed Kähler manifolds, Int. Math. Res. Not., posted on 2006, Art. ID 63640, 18, DOI 10.1155/IMRN/2006/63640. MR:2233716 (2007b:32058)

[22] Zhou Zhang, Degenerate Monge-Ampere equations over projective manifolds, ProQuest LLC, Ann Arbor, MI, 2006. Thesis (Ph.D.)-Massachusetts Institute of Technology. MR2717347

School of Mathematics and Statistics, University of Sydney, NSW 2006, Australia E-mail address: zhangou@maths.usyd.edu.au 